\title{
Combined gefitinib and pemetrexed overcome the acquired resistance to epidermal growth factor receptor tyrosine kinase inhibitors in non-small cell lung cancer
}

\author{
MIN WU ${ }^{1}$, YUAN YUAN ${ }^{2}$, YUE-YIN PAN ${ }^{3}$ and YING ZHANG ${ }^{1}$ \\ ${ }^{1}$ Department of Geriatrics; ${ }^{2}$ The Central Laboratory of Binhu Hospital, \\ The Third Affiliated Hospital of Anhui Medical University, Hefei, Anhui 230061; ${ }^{3}$ Department of Oncology, \\ The First Affiliated Hospital of Anhui Medical University, Hefei, Anhui 230022, P.R. China
}

Received August 11,2013; Accepted April 2, 2014

DOI: $10.3892 / \mathrm{mmr} .2014 .2243$

\begin{abstract}
Currently, chemotherapy and targeted therapies share the principal limitation of the emergence of drug resistance, which prevents these strategies from having lasting clinical benefits. The combination of epidermal growth factor receptor tyrosine kinase inhibitors (EGFR-TKIs) with concurrent chemotherapy has been proposed as one strategy to overcome acquired resistance to EGFR-TKIs. The purpose of the present study was to investigate the combined effects of gefitinib and pemetrexed on EGFR-TKI-sensitive and EGFR-TKI-resistant human non-small cell lung cancer (NSCLC) cell lines. The antiproliferative effects of gefitinib and pemetrexed, alone and in combination, on the growth of NSCLC cell lines, were assessed using an MTT assay. The cytotoxic interaction between the two drugs was evaluated in vitro using the combination index (CI) method. Cell cycle distribution and apoptosis were analyzed by flow cytometry and alterations in signaling pathways were determined by western blot analysis. In the present study, it was identified that when cells were concurrently exposed to pemetrexed and gefitinib, cytotoxic synergism was present in the gefitinib-resistant PC9/GR human NSCLC cell line and antagonistic interactions were observed in the gefitinib-sensitive PC9 cell line. Synergism was associated with a combination of cell cycle effects of the different agents. In addition, the combination of pemetrexed and gefitinib decreased the levels of phosphorylated AKT, phosphorylated
\end{abstract}

Correspondence to: Professor Yue-Yin Pan, Department of Oncology, The First Affiliated Hospital of Anhui Medical University, Ji Xi Road, Hefei, Anhui 230022, P.R. China

E-mail: panyueyin1965@126.com

Professor Ying Zhang, Department of Geriatrics, The Third Affiliated Hospital of Anhui Medical University, 390 Huai He Road, Hefei, Anhui 230061, P.R. China

E-mail: yingzhang1965@163.com

Key words: gefitinib, pemetrexed, non-small cell lung cancer, acquired resistance, combination treatment extracellular-signal-regulated kinase and B-cell lymphoma 2 as compared with those in the control. By contrast, antagonism was associated with gefitinib-induced G0/G1-phase blockade of gefitinib-sensitive cells, which interfered with the cell cycle-specific cytotoxicity of chemotherapy. The combination of pemetrexed and gefitinib generated synergistic effects in gefitinib-acquired resistant cells and antagonistic effects in gefitinib-sensitive cells, suggesting that EGFR-TKIs combined with pemetrexed may be a beneficial treatment strategy for NSCLC patients with acquired resistance to EGFR-TKIs.

\section{Introduction}

Lung cancer is the leading cause of cancer-associated mortality worldwide and non-small cell lung cancer (NSCLC) represents $\sim 80-85 \%$ of all lung cancers (1). Unfortunately, the majority of patients are diagnosed at an advanced stage, when drug therapy is the main treatment option. Although systemic chemotherapy improves the lung cancer survival rate, treatment outcomes for advanced NSCLC remain unsatisfactory.

Epidermal growth factor receptor (EGFR) belongs to the ErbB family of plasma membrane receptor tyrosine kinases and is unduly expressed in numerous cancer types, including $40-80 \%$ of NSCLCs $(2,3)$. Patients whose tumors harbor somatic-activating mutations within the EGFR gene greatly benefit from treatment with EGFR-tyrosine kinase inhibitors (EGFR-TKIs) (4). The recent development of anticancer drugs that target EGFR has improved the survival rates and triggered a paradigm shift for the treatment of NSCLC patients $(5,6)$.

However, all patients with EGFR mutant tumors will inevitably develop progressive disease following 10-14 months of EGFR-TKI treatment, a concept referred to clinically as acquired resistance (7). Currently, novel treatment strategies are urgently required to overcome acquired resistance to EGFR-TKIs. The combination of continued EGFR-TKIs with concurrent chemotherapy has been proposed as a strategy to overcome acquired resistance (8). A recent retrospective analysis demonstrated improved response rates (RR) when EGFR-TKIs were continued with chemotherapy, following evident disease progression on the TKIs alone (9). Additionally, 
several strategies combining chemotherapy with continued EGFR-TKIs at the time of progression have been put forward.

Preclinical and clinical studies suggested that the synergy of targeted agents with conventional cytotoxic agents may not only have lower toxicity but also be an improved, more effective cancer treatment option (10). Gefitinib, erlotinib, docetaxel and pemetrexed have been widely used for second-line treatment of advanced NSCLC. Gefitinib and pemetrexed have distinctly improved tolerability and lower toxicity compared with docetaxel, and their use has promptly increased as a result $(11,12)$.

Gefitinib is a tyrosine kinase inhibitor (TKI) that inhibits the EGFR through competitive inhibition of the ATP-binding site in the tyrosine kinase domain (13). It has been approved for its clinical benefit in the treatment of advanced NSCLC patients who have previously received platinum-based chemotherapy (14). It inhibits the activation of ERK and AKT and induces growth arrest of tumor cells (15). Gefitinib causes cell growth arrest in G0/G1 phase and induces apoptosis in a variety of tumor cell types $(16,17)$. EGFR-TKIs have demonstrated promising synergistic effects in combination with various cytotoxic drugs against various human tumor types in vitro and in vivo (18-20).

Pemetrexed is a multitargeted antifolate cytotoxic agent which potently inhibits multiple key enzymes, including thymidylate synthase (TS), dihydrofolate reductase (DHFR) and glycinamide ribonucleotide formaldehyde transferase (GARFT) (21). Pemetrexed arrests cells mainly in the S phase of the cell cycle and induces apoptosis in various solid tumor types, including NSCLC (22). Phase III clinical trials demonstrated that pemetrexed has equal efficacy and milder toxicity and improved safety profiles compared with previous standard cytotoxic drugs in first- and second-line treatment $(23,24)$.

According to the above information, it was hypothesized that the combination of an EGFR-TKI and a chemotherapeutic agent may represent a more effective therapeutic strategy for NSCLC patients who are resistant to EGFR-TKI therapy alone. In the present study, the effects of gefitinib in combination with pemetrexed were examined in gefitinib-sensitive and gefitinib-acquired resistant NSCLC cell lines in vitro.

\section{Materials and methods}

Drugs. Gefitinib (IRESSA) was obtained from AstraZeneca (London, England, UK) and was dissolved in dimethylsulfoxide (DMSO; Sigma, St. Louis, MO, USA) to a stock concentration of $10 \mathrm{mmol} / \mathrm{l}$. Pemetrexed (Alimta; Eli Lilly, Indianapolis, IN, USA) was obtained commercially from the pharmacy at The Third Affiliated Hospital of Anhui Medical University (Hefei, China) and was dissolved in $0.9 \% \mathrm{NaCl}$ to a final concentration of $10 \mathrm{mmol} / \mathrm{l}$. The two drugs were stored at $-20^{\circ} \mathrm{C}$ in tightly sealed sterile tubes and diluted to the desired concentrations in culture medium prior to use. The final concentration of DMSO in Dulbecco's modified Eagle's medium (DMEM; Hyclone, Logan, UT, USA) was kept at $<0.1 \%$ and equal amounts of the solvent were added to the control cells.

Cell culture. The EGFR-TKI-sensitive human NSCLC cell line PC9 and the gefitinib-acquired-resistant cell line PC9/GR were provided by Guangdong Lung Cancer Institute (Guangdong, China) and were cultured in DMEM supplemented with $10 \%$ heat-inactivated fetal bovine serum (Hyclone), penicillin $(100 \mathrm{U} / \mathrm{ml})$, streptomycin $(100 \mu \mathrm{g} / \mathrm{ml})$ and L-glutamine $(2 \mathrm{mM})$ in a humidified atmosphere with $5 \% \mathrm{CO}_{2}$ at $37^{\circ} \mathrm{C}$ and then harvested with trypsin-EDTA when the cells reached exponential growth.

Growth inhibition assay. The antiproliferative effects of gefitinib and pemetrexed as single agents on cells were evaluated by an MTT assay. Exponentially growing cells were seeded in 96-well plastic plates at a density of 3,500 and 4,000 cells/well for PC9 and PC9/GR, respectively. Cells were then cultured overnight and treated with various concentrations of gefitinib and pemetrexed for $72 \mathrm{~h}$. Following exposure to each drug for $72 \mathrm{~h}$ in 96 -well plates, $20 \mu \mathrm{l}$ MTT solution was added to each well and cells were incubated for $4 \mathrm{~h}$ at $37^{\circ} \mathrm{C}$. The colored formazan product was then dissolved by adding $150 \mu \mathrm{l}$ DMSO. The 96-well plates were placed on a shaker for $10 \mathrm{~min}$ at room temperature to thoroughly dissolve the formazan product. Then the optical density (OD) of each well was measured at $490 \mathrm{~nm}$ on an ELISA plate reader (Bio-Rad Laboratories, Inc. Winooski, VT, USA). The percentage of cell growth inhibition resulting from each drug was calculated as: [(OD490 control cells $_{-}$OD490 cells $\left.) / \mathrm{OD} 490_{\text {control cells }}\right] \times 100 \%$. The $\mathrm{IC}_{50}$-value was the concentration resulting in $50 \%$ cell growth inhibition by a $72 \mathrm{~h}$ exposure to the drug(s) compared with the untreated control cells. Six replicate wells were used for each drug concentration and the testing was conducted independently at least three times.

Drug combination studies. The antiproliferative effects of the interaction between gefitinib and pemetrexed were evaluated by measuring the combination index $(\mathrm{CI})$, a quantitative representation of pharmacological interactions between two drugs. The combination drug doses for constant ratios of the $\mathrm{IC}_{50}$-values were calculated from the previous growth inhibition assay. Thus, the CI-value was calculated using 0.125 , $0.25,0.5,1,2$ and 4 times the $\mathrm{IC}_{50}$ of gefitinib and pemetrexed combination doses. The CI-values of interactions between gefitinib and pemetrexed were analyzed according to the Chou and Talaly method using the CompuSyn software (ComboSyn, Inc., Paramus, NJ, USA). CI $<1, \mathrm{CI}=1$ and $\mathrm{CI}>1$ indicate a synergistic, additive and antagonistic effect, respectively (25).

Cell cycle analysis by flow cytometry. Cells (1x10\% $/$ well) were plated in six-well dishes and treated with gefitinib and pemetrexed as single agents and in combination at the concentration of the $\mathrm{IC}_{50}$-value for $72 \mathrm{~h}$ as described above. At the end of each exposure, the adhered cells were harvested by trypsinization, washed twice with phosphate-buffered saline (PBS) and fixed with $75 \%$ cold ethanol at $4^{\circ} \mathrm{C}$ overnight. Following removal of the ethanol, the cells were washed twice in PBS and then resuspended in $1 \mathrm{ml}$ of propidium iodide (PI)/Triton X-100 staining solution [PBS containing 0.1\% Triton X-100 (Sigma), $200 \mu \mathrm{g} / \mathrm{ml}$ RNAse A (Sigma) and $50 \mu \mathrm{g} / \mathrm{ml}$ PI (Sigma)] in the dark for $30 \mathrm{~min}$. The cell cycle was assessed by flow cytometry and the percentage of cells in G1, S and G2/M phases of the cell cycle were calculated using ModFit LT ${ }^{\mathrm{TM}}$ software, version 4.0 (Verity Software House, Topsham, ME, USA).

Flow cytometric analysis of apoptosis. Cells in the exponential growth phase were plated in six-well plates, allowed to attach 
A

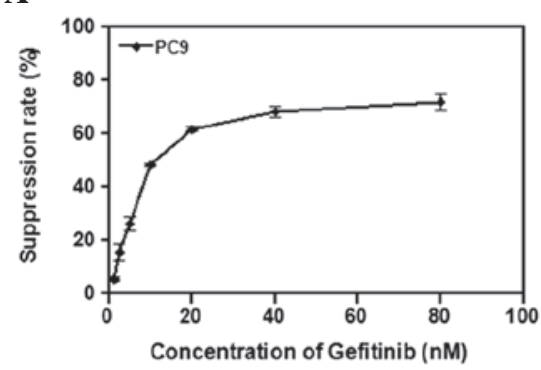

B

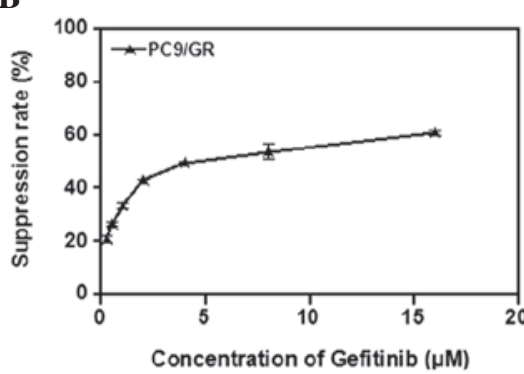

C

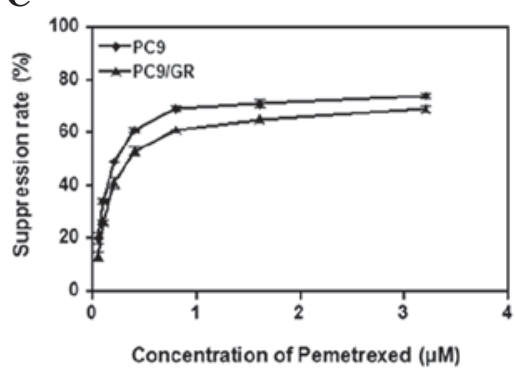

Figure 1. In vitro effects of gefitinib and pemetrexed on proliferation of NSCLC cell lines. MTT assays were used to examine the inhibitory activities on cell proliferation. The cells were exposed to varying concentrations of gefitinib in (A) PC9 (1.25-80 nmol/1) and (B) PC9/GR cells (0.25-16 $\mu$ mol/1) or (C) pemetrexed in the cells $(0.05-3.2 \mu \mathrm{mol} / \mathrm{l})$ for $72 \mathrm{~h}$. Each data point was repeated in at least three independent experiments. NSCLC, non-small cell lung cancer.

overnight and treated with gefitinib and pemetrexed alone or in combination using the concentration of the $\mathrm{IC}_{50}$-values for $72 \mathrm{~h}$. Following $72 \mathrm{~h}$ of treatment, adherent and floating cells were collected, washed twice with pre-cooled $\left(4^{\circ} \mathrm{C}\right) \mathrm{PBS}$ and resuspended in $400 \mu \mathrm{l}$ binding buffer. Cells were first incubated with $5 \mu \mathrm{l}$ Annexin V-fluorescein isothiocyanate (FITC) at room temperature in the dark for $15 \mathrm{~min}$ and then with $10 \mu \mathrm{l}$ PI $(40 \mu \mathrm{g} / \mathrm{ml})$ at room temperature in the dark for $5 \mathrm{~min}$. Cell suspensions were transferred to flow cytometry test tubes and detected by flow cytometry. Cells with no drug treatment were used as a control. Data were analyzed by CellQuest software (Becton Dickinson, San Jose, CA, USA)

Western blot analysis. Cells were seeded at a density of $6 \times 10^{5}$ in $100 \mathrm{~mm}^{2}$ dishes for $24 \mathrm{~h}$ prior to treatment. Following $72 \mathrm{~h}$ of incubation with single or double drug combinations, cells were washed with ice-cold PBS solution and scraped in lysis buffer. The lysates were centrifuged at 13,380 x g for $30 \mathrm{~min}$ at $4^{\circ} \mathrm{C}$ and then the supernatant was collected. Equivalent amounts of proteins were analyzed by SDS-PAGE and transferred to PVDF membranes. Appropriate primary antibodies to p-AKT, AKT, phosphorylated extracellular-signal-regulated kinase (p-ERK), ERK, B-cell lymphoma 2 (Bcl-2) and $\beta$-actin purchased from Cell Signaling Technology, Inc. (Beverly, MA, USA) were used. Proteins were visualized with a horseradish peroxidase-coupled secondary antibody from Cell Signaling Technology, Inc. Positive bands were detected using enhanced chemiluminescence reagents (Millipore, Billerica, MA, USA). $\beta$-actin was used as a loading control. The band densities were scanned for densitometric analysis using ImageJ 1.43 software (NIH Image, Bethesda, MD, USA).

Statistical analysis. All data were assayed in three independent experiments. The results were presented as the mean \pm standard deviation. Student's t-test and one-way analysis of variance were used to assess the statistical significance between values and a level of $\mathrm{P}<0.05$ was considered to indicate a statistically significant difference.

\section{Results}

Dose-dependent antiproliferative effects of gefitinib and pemetrexed in human NSCLC cell lines. The antiproliferative activity of gefitinib and pemetrexed as single agents on PC9 and PC9/GR cells were evaluated using an MTT assay.
The cells were exposed to the varying concentrations of gefitinib (1.25-80 nmol/l for PC9 and $0.25-16 \mu \mathrm{mol} / 1$ for PC9/GR cells) or pemetrexed (0.05-3.2 $\mu \mathrm{mol} / 1$ for both cell lines) for 72 h. Fig. 1 illustrates the growth-inhibitory effect of gefitinib on the parent PC9 cell line and its resistant subline, PC9/GR. The $\mathrm{IC}_{50}$-value of gefitinib in PC9 cells was $0.016 \mu \mathrm{mol} / \mathrm{l}$, as compared with $4.94 \mu \mathrm{mol} / \mathrm{l}$ in PC9/GR cells (306-fold resistance). Dose-dependent growth inhibition by pemetrexed was observed in the two NSCLC cell lines, where PC9/GR cells exhibited no cross resistance to pemetrexed, with $\mathrm{IC}_{50}$-values of $<1 \mu \mathrm{mol} / 1$ in all cases (Table I). This concentration is markedly lower than the mean peak plasma concentration of pemetrexed achievable in patients (i.e., 120-230 $\mu \mathrm{mol} / \mathrm{l}$ ), indicating a unexpectedly high sensitivity of NSCLC cells to this agent in vitro $(26,27)$.

Effects of combined gefitinib and pemetrexed treatment in human EGFR-TKI-sensitive and EGFR-TKI-resistant cells. The interaction between gefitinib and pemetrexed on PC9 and PC9/GR cell lines was evaluated. They were exposed to various concentrations of gefitinib and pemetrexed concomitantly for 72 h. As illustrated in Fig. 2, the PC9/GR cells, when treated with gefitinib and pemetrexed concurrently, CI-values were all $<1$, with mean CI-values of 0.59 , suggesting a synergistic interaction between gefitinib and pemetrexed in cells with acquired EGFR-TKI resistance. However, similar results were not identified in PC9 cells. In EGFR-TKI-sensitive PC9 cells, concurrent administration resulted in antagonistic effects (CI $>1$ ), with mean CI-values of 1.24 (Fig. 2).

Effects of pemetrexed and gefitinib on the cell cycle. The cell cycle distribution was analyzed in cells exposed to pemetrexed or gefitinib alone or concurrently for $72 \mathrm{~h}$ by flow cytometry. The cell cycle effects of different exposures as compared with the unexposed control cells are demonstrated in Fig. 3. As illustrated in Fig. 3, in the EGFR-TKI-sensitive PC9 cells, when treated with gefitinib alone, there was a significant cell cycle arrest at the G0/G1-phase $(\mathrm{P}<0.01)$, while in EGFR-TKIresistant PC9/GR cells, when administered with gefitinib alone, there was no evident additional G0/G1-phase arrest. Pemetrexed alone induced S-phase arrest in $\sim 41 \%$ of PC9/GR cells $(\mathrm{P}<0.01)$ and resulted in $\mathrm{S}$-phase arrest in $\sim 44 \%$ of $\mathrm{PC} 9$ cells $(\mathrm{P}<0.01)$. Concurrent exposure of PC9 cells to pemetrexed and gefitinib resulted in a similar cell cycle arrest pattern (mainly G1-phase arrest) to that observed with gefitinib administered alone and a 
Table $\mathrm{I} \mathrm{IC}_{50}$-values of gefitinib and pemetrexed were determined by the MTT assay.

\begin{tabular}{lcc}
\hline $\mathrm{IC}_{50}$ & PC9 $(\mathrm{nM})$ & PC9/GR $(\mu \mathrm{M})$ \\
\hline Pemetrexed & $292.66 \pm 26.01$ & $0.51 \pm 0.023$ \\
Gefitinib & $16.05 \pm 1.85$ & $4.94 \pm 0.440$ \\
\hline
\end{tabular}

$\mathrm{IC}_{50}$, concentration resulting in inhibition of $50 \%$ of the maximal cell growth.

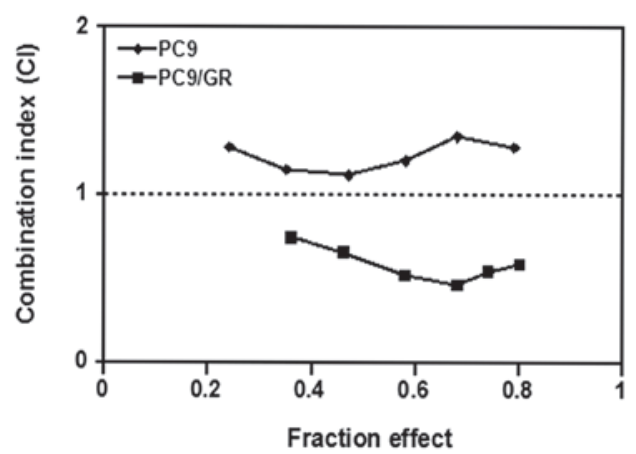

Figure 2. The CI value was calculated using the Chou-Talalay method following the combination of gefitinib with pemetrexed treatment as described previously. $\mathrm{CI}>1$ in the PC9 cell line; $\mathrm{CI}<1$ in the PC9/GR cell line. $\mathrm{CI}<1$, $\mathrm{CI}=1$ and $\mathrm{CI}>1$ indicate synergistic, additive and antagonistic effects, respectively. CI, combination index.

corresponding reduction of arrest in $\mathrm{S}$-phase $(\mathrm{P}<0.05)$, which prevented the S-phase arrest effect of pemetrexed. By contrast, when PC9/GR cells were exposed to both drugs, alterations in the cell cycle phase distribution and overlapping effects of pemetrexed and gefitinib were observed, which may amplify the cytotoxicity of pemetrexed and gefitinib.

Effects of pemetrexed or gefitinib alone or in combination on cell apoptosis. To examine whether the observed growth inhibition was due to enhanced apoptosis, the rates of apoptosis of each single or combined drug treatment in EGFR-TKI-sensitive and EGFR-TKI-resistant cell lines were evaluated using the concentration of the $\mathrm{IC}_{50}$-value for each drug with subsequent analysis using an Annexin V/PI assay. As demonstrated in Fig. 4, the rates of apoptosis induced by pemetrexed in PC9 and PC9/GR after $72 \mathrm{~h}$ were 14.54 and $19.54 \%$, respectively. Treatment with gefitinib alone for $72 \mathrm{~h}$ resulted in the apoptotic rates of 9.6 and $7.64 \%$ in PC9 and PC9/GR cells, respectively. However, exposure to pemetrexed combined with gefitinib resulted in a decrease in the induction of apoptosis in $11.68 \%$ of PC9 cells. By contrast, the rates of apoptosis induced by concurrent exposure in PC9/GR cells after $72 \mathrm{~h}$ were significantly increased to $35.88 \%$. These data indicated that concurrent exposure to pemetrexed and gefitinib resulted in a negative interaction in gefitinib-sensitive cells and a synergistic interaction in gefitinib-resistant NSCLC cells.

Gefitinib or pemetrexed alone or in combination modify expression levels of EGFR signaling-associated proteins. ERK and AKT are important downstream targets of the
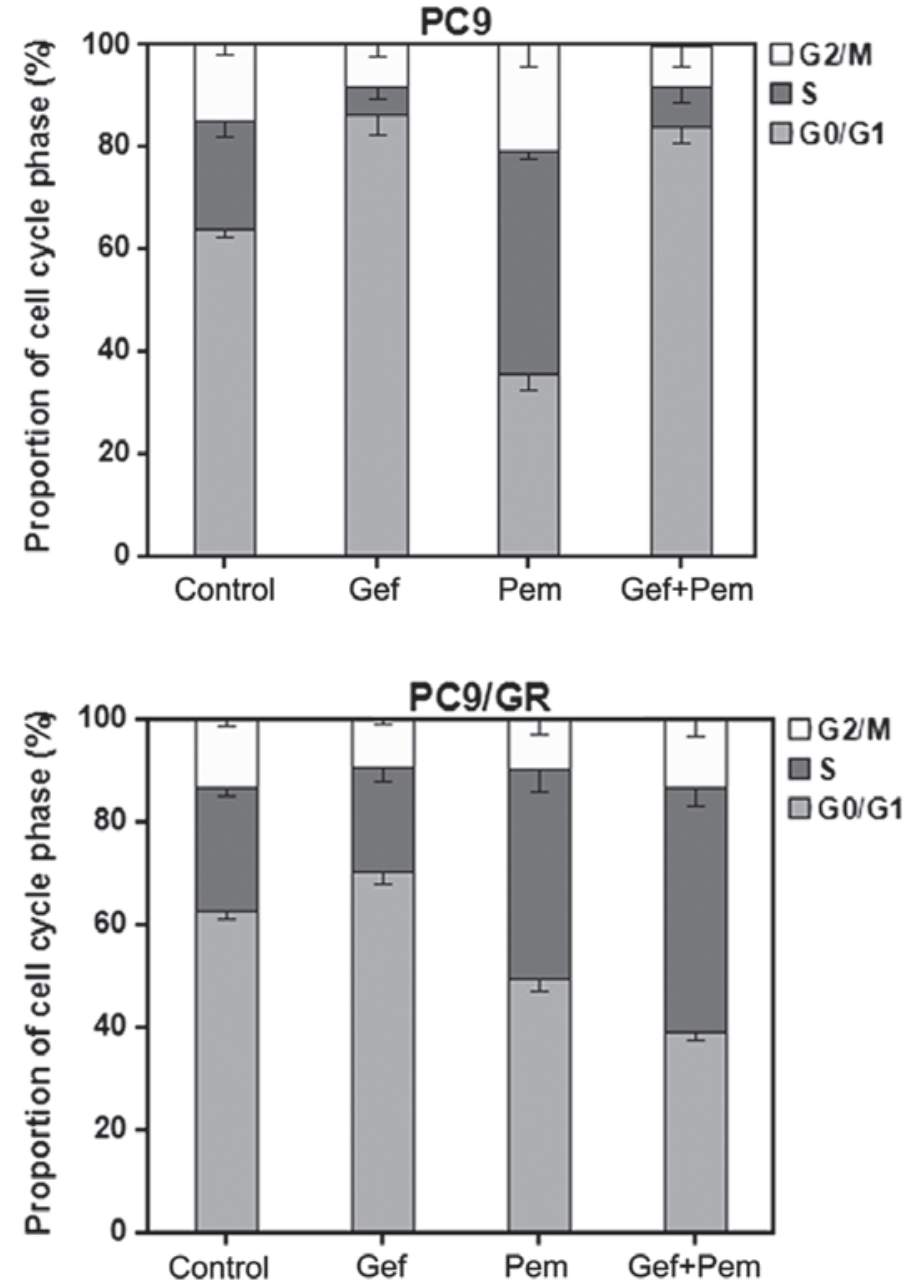

Figure 3. Flow cytometric analysis was utilised to determine alterations in cell cycle distributions in NSCLC cell lines after administration of pemetrexed and gefitinib as single agents or concurrently for $72 \mathrm{~h}$. Pemetrexed and gefitinib were used at their respective $\mathrm{IC}_{50}$ concentrations. Gef, Pem, and Gef + Pem refer to gefitinib, pemetrexed and concurrent administration, respectively. NSCLC, non-small cell lung cancer.

EGFR pathway. To further elucidate the potential mechanisms involved in regulating the interaction between pemetrexed and gefitinib, western blot analysis was used to evaluate the effects of single or combined drugs on the EGFR downstream signaling pathway, the levels of phosphorylated ERK and phosphorylated AKT in gefitinib-sensitive and gefitinib-resistant cells. As illustrated in Fig. 5, gefitinib inhibited the activation of the EGFR downstream signaling mediators ERK and AKT effectively in gefitinib-sensitive PC9 cells, whereas the inhibitory effects of gefitinib on these signaling pathways in gefitinib-resistant PC9/GR cells were significantly less than those in parent PC9 cells. These results suggested that downregulation of activated AKT and ERK is correlated with cellular sensitivity to gefitinib. Following $72 \mathrm{~h}$ of exposure to pemetrexed at concentrations of the $\mathrm{IC}_{50}$-values, the levels of p-ERK and p-AKT were upregulated in PC9/GR cells as compared with untreated cells. By contrast, in the PC9 cells, exposure to pemetrexed did not result in increased p-AKT and p-ERK levels.

In addition, it was identified that when the PC9/GR cells were exposed to a combination of pemetrexed and gefitinib for $72 \mathrm{~h}$, the 

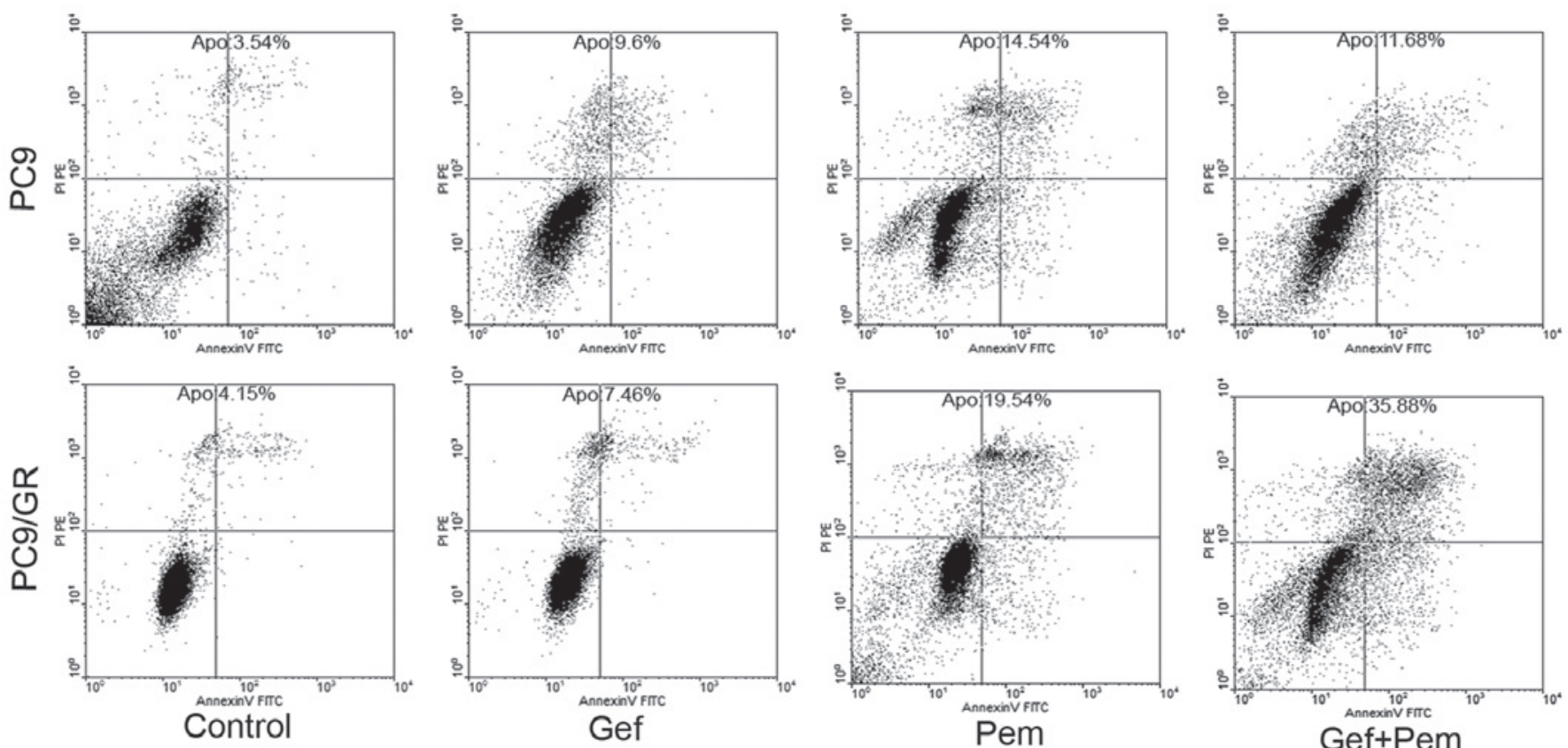

Figure 4. Effect of gefitinib and pemetrexed on cell apoptosis. The effects of each single drug and their combination on apoptosis were analyzed in PC9 and PC9/GR cell lines using flow cytometry. Following $72 \mathrm{~h}$ of treatment, adherent and floating cells were collected and incubated with Annexin V and propidium iodide. Upper left quadrants $\left(\mathrm{An}^{-} / \mathrm{PI}^{+}\right)$, necrotic cells; upper right quadrants $\left(\mathrm{An}^{+} / \mathrm{PI}^{+}\right)$, late apoptotic cells; lower left quadrant $(\mathrm{An} / \mathrm{PI})$, live cells; and lower right quadrant $\left(\mathrm{An}^{+} / \mathrm{PI}^{-}\right)$, early apoptotic cells. Pem, Gef and Gef + Pem refer to pemetrexed, gefitinib and concurrent administration, respectively.

A

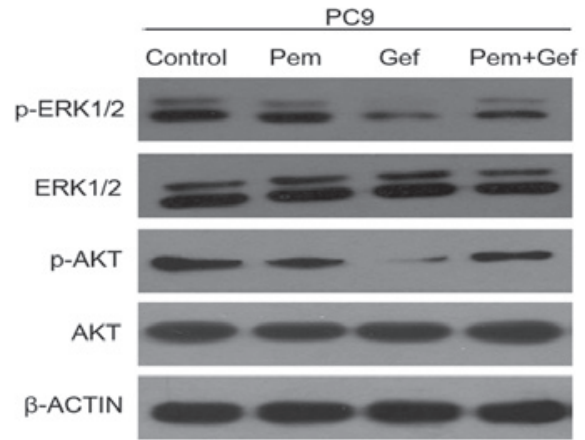

C

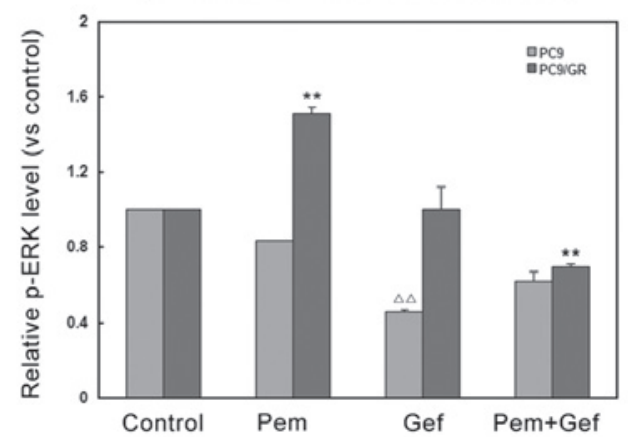

B

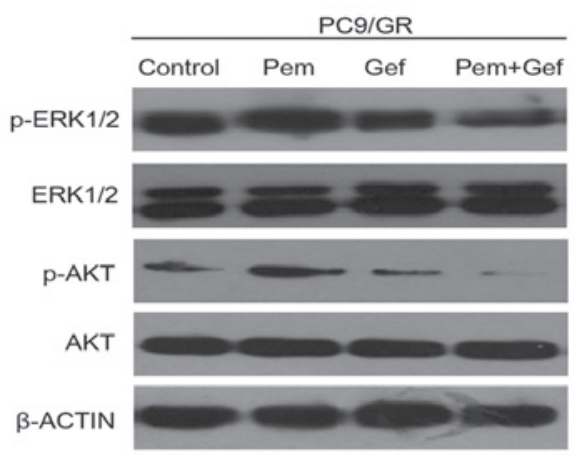

D

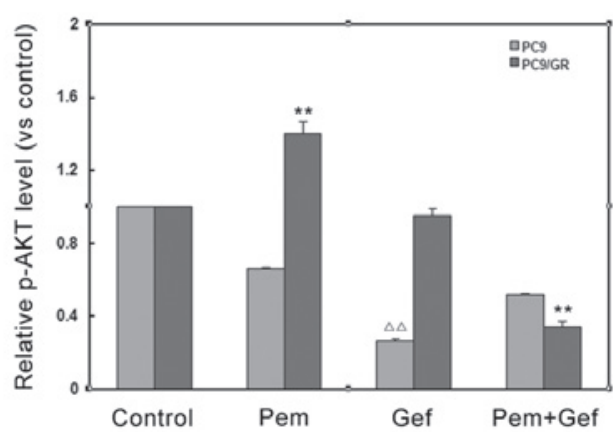

Figure 5. Effects of gefitinib and pemetrexed alone and in concurrent exposure for $72 \mathrm{~h}$ at their $\mathrm{IC}_{50}$ concentration. Proteins of downstream signaling pathways were assessed by western blot analysis with the corresponding antibodies. $\beta$-actin was used as loading control. The expression levels of the proteins were detected in (A) PC9 and (B) PC9/GR cells. (C and D) The relative phosphorylated ERK and phosphorylated AKT levels compared with the control. ${ }^{* *} \mathrm{P}<0.01$ compared with PC9/GR control. ${ }^{\triangle} \triangle \mathrm{P}<0.01$ compared with PC9 control. Pem, Gef and Gef + Pem refer to pemetrexed, gefitinib and concurrent administration, respectively. pERK, phosphorylated extracellular-signal-regulated kinase.

levels of p-AKT and p-ERK were decreased compared with the levels observed in the control or single-agent treatment, whereas, in the PC9 cells, the combination of the two drugs increased the levels of p-AKT and p-ERK. However, there was no significant variation in the total ERK and AKT expression levels compared with the control (Fig. 5). These results suggested that although gefitinib alone did not inhibit p-AKT and p-ERK1/2 expression, as was expected in the gefitinib-resistant cells, gefitinib blocked the pemetrexed-induced activation of phosphorylated ERK and phosphorylated AKT when exposed concurrently. 


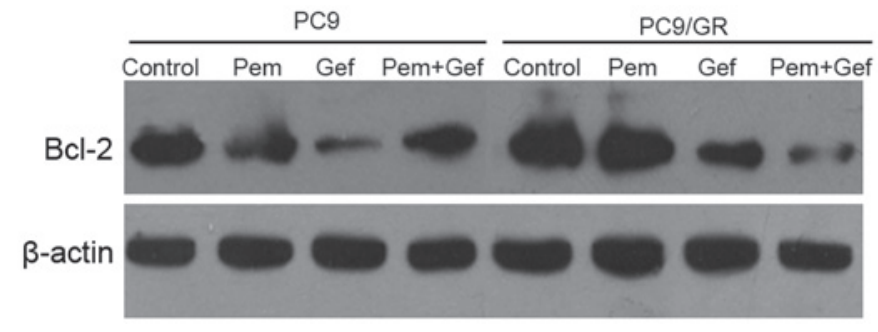

Figure 6. Combination effects of pemetrexed and gefitinib on Bcl-2 protein expression in PC9 and PC9/GR cells. Cells were treated with gefitinib, pemetrexed and their combinations for $72 \mathrm{~h}$ using their $\mathrm{IC}_{50}$ concentrations. Following treatment, cells were harvested and lysed, and equal aliquots of extracted protein were analyzed for Bcl-2 expression by western blot analysis. Pem, Gef and Pem + Gef refer to pemetrexed, gefitinib and concurrent administration, respectively. The levels of $\beta$-actin served as the internal control. Bcl-2, B-cell lymphoma 2.

Effects of gefitinib and pemetrexed treatment on Bcl-2 levels. To further elucidate the mechanisms of cell death induced by treatment with pemetrexed and gefitinib, the expression of $\mathrm{Bcl}-2$ was detected by western blot analysis. As illustrated in Fig. 6, it was identified that the basal levels of Bcl-2 were higher in PC9/GR cells than those in PC9 cells and the inhibitory effect of gefitinib on Bcl-2 in PC9/GR cells was noticeably lower than that in PC9 cells. When the two cell lines were exposed to the concurrent treatment, the levels of Bcl-2 were effectively downregulated in gefitinib-resistant cells, while in gefitinib-sensitive PC9 cells, upregulation of Bcl-2 expression levels was observed as compared with the single-agent treatment.

\section{Discussion}

Currently, platinum-based chemotherapy is considered to be the mainstay of first-line treatment for advanced NSCLC (28). Despite the optimization of chemotherapeutic strategies, there have been no second-line combination chemotherapy regimens with a proven a survival benefit, due to excessive toxicities and the rapidly declining clinical condition of patients $(29,30)$. Recently, targeted anticancer drugs, including the EGFR-TKIs gefitinib and erlotinib, have been approved for the treatment of lung cancer (31). However, although targeted therapies have been successfully developed, only a small subset of NSCLC patients with EGFR mutant tumors benefit from the EGFR-TKIs, and patients who initially respond to the treatment often ultimately develop acquired resistance to these targeted therapies (32). Therefore, the development of new treatment strategies for NSCLC to overcome acquired resistance to EGFR-TKIs is urgently required.

A key issue in the novel treatment modalities against advanced NSCLC is the integration of EGFR-TKIs with chemotherapy. Preclinical studies have revealed that coadministration with EGFR-TKIs enhanced the effect of different cytotoxic agents against various tumor models, including NSCLC cells $(33,34)$. However, there have been few studies that used combinations of chemotherapy and targeted anticancer agents in the treatment of NSCLCs with acquired EGFR-TKI resistance.

PC9 is a lung adenocarcinoma cell line, which has a deletion mutation within the kinase domain of EGFR and is highly sensitive to EGFR-TKIs. These characteristics are similar to those of NSCLC with clinical responsiveness to gefitinib.
The PC9/GR subline is specifically resistant to gefitinib, which is similar to that of patients with acquired resistance to EGFR-TKIs. In the present study, the antiproliferative effects of gefitinib, as a single agent and in combination with pemetrexed in the EGFR-TKI-sensitive PC9 cell line and its resistant subline, PC-9/GR, was investigated.

It was identified that gefitinib and pememtrexed exhibited dose-dependent growth inhibition when used as single agents in the two lung cancer cells, but in PC9/GR cells, significant antiproliferative effects of gefitinib were observed only at higher concentrations. The $\mathrm{IC}_{50}$-values of gefitinib to PC9/GR cells were higher than those to PC9 cells. These results evidently demonstrated the significant in vitro resistance of PC9/GR cells to gefitinib. Synergism was observed when gefitinib was combined with pemetrexed in the PC9/GR cells, whereas antagonistic interactions were noted in the PC9 cells.

Previous studies have demonstrated controversial results regarding the concurrent antiproliferative effects in EGFR-TKI-sensitive cell lines $(34,35)$. The discrepancies in the antiproliferative effects of combined administration may be in part due to the different heritage characteristics of the cell lines and different drug exposure conditions. Therefore, patients may benefit from the concomitant administration of pemetrexed and gefitinib, particularly in those with acquired resistance to EGFR-TKIs. In addition, the results of the present study also demonstrated consistent results in the rate of apoptosis.

The mechanisms of these synergistic and antagonistic effects may be explained by differences in the changes induced in the cell cycle. In the present study, pemetrexed arrested the cell cycle at the S-phase in the cell lines, whereas gefitinib caused significant G0/G1 phase accumulation only in the PC9 cell line. When exposed to a combination of pemetrexed and gefitinib, the proportion of cells in $\mathrm{S}$ phase increased compared with the control in the PC9/GR cells. Due to the fact that gefitinib was not able to induce additional G1-phase arrest in PC9/GR cells with acquired resistance to gefitinib, continuous daily administration of gefitinib is expected to have a minimal negative effect on the cytotoxicity of pemetrexed. However, in PC9 cells, concurrent exposure resulted in a mainly G0/G1-phase arrest, which is similar to gefitinib administered alone. The mechanisms underlying the observed antagonistic effects of concurrent administration of EGFR-TKIs and chemotherapy are relevant to pharmacodynamic separation, since G0/G1-phase arrest induced by EGFR-TKIs in NSCLC possibly interferes with the cell cycle-specific cytotoxicity of chemotherapy, resulting in decreased cytotoxicity (36).

By contrast, part of this growth inhibition may be explained by apoptosis. Bcl-2 is an anti-apoptotic member of the Bcl-2 family, which is a key apoptosis regulator that is downregulated in numerous cell types in response to treatment leading to activation of apoptosis $(37,38)$. The present study demonstrated that combination treatment of gefitinib and pemetrexed resulted in high levels of Bcl-2 activation in gefitinib-sensitive PC9 cells, whereas downregulation of the anti-apoptotic protein Bcl-2 was observed in PC9/GR cells. These results indicated that downregulation of the antiapoptotic protein Bcl-2 may be involved in combination-induced cell death.

To elucidate the mechanism underlying the different antiproliferative effects of gefitinib combined with peme- 
trexed, drug-induced growth signaling pathway expression levels following pemetrexed exposure were evaluated. These results demonstrated that pemetrexed increased the levels of p-ERK and p-AKT in the gefitinib-resistant PC9/GR cells. By contrast, in the PC9 cells, pemetrexed exposure did not result in increased p-AKT and p-ERK levels. Following this, a possible association between sensitivity to gefitinib and inhibition of EGFR downstream signaling molecules p-AKT and p-ERK was evaluated. Previous studies have demonstrated that inhibition of PI3K/AKT activity correlates with the sensitivity to EGFR-TKIs in NSCLC cells $(39,40)$. Consistent with this, the present study identified that in the PC9 cell line, which is highly sensitive to gefitinib, there was a strong inhibition of p-AKT and $\mathrm{p}$-ERK following treatment with gefitinib. However, there was no inhibition of p-AKT and p-ERK following treatment with gefitinib in the gefitinib-resistant PC9/GR cell line. These data suggested that in the gefitinib-sensitive NSCLC cell lines, the AKT and ERK pathways are more dependent on signals transduced by EGFR than is the case in the gefitinib-resistant NSCLC cell lines.

When gefitinib was combined with pemetrexed, a significant decrease in p-AKT and p-ERK was observed in the PC9/GR cells, as compared with the control. However, the levels of p-AKT and p-ERK increased when gefitinib and pemetrexed were applied together to the PC9 cells. The results of the present study suggested that only NSCLC tumors that respond to pemetrexed by upregulating p-AKT and p-ERK will respond to the addition of EGFR-targeted TKIs. Furthermore, a number of previous studies have demonstrated similar results: Only cell lines that responded with increased EGFR activation following chemotherapy were synergistically growth inhibited following the addition of gefitinib to chemotherapy $(33,41)$. These effects on p-AKT and p-ERK in NSCLC cells may explain the synergistic or antagonist growth inhibitory effects observed in the two cell lines treated with gefitinib and pemetrexed.

In conclusion, the present study demonstrated the effect of in vitro single-agent gefitinib and concurrent growth inhibitory effects of gefitinib and pemetrexed in the EGFR-TKI-sensitive and the EGFR-TKI-resistant NSCLC cell lines. It was identified that gefitinib combined with pemetrexed generated synergistic effects in cells with acquired gefitinib resistance and antagonistic effects in gefitinib-sensitive cells. The present study suggested that EGFR-TKIs combined with pemetrexed may be beneficial to NSCLC patients with acquired EGFR-TKI resistance. The ongoing IMPRESS (NCT01544179) randomized phase III trial, which evaluates continued administration of gefitinib with the addition of chemotherapy, compared with chemotherapy alone in EGFR mutation-positive patients who have progressed on first-line gefitinib, will indicate whether this preclinical observation is clinically relevant or of therapeutic value.

\section{Acknowledgements}

This study was supported by a grant from the Anhui Provincial Natural Science Research Program of Higher Education Institutions Foundation of China (no. KJ2012A157) and by The Central Laboratory of The Third Affiliated Hospital of Anhui Medical University. The authors are grateful to Dr Xuchao Zhang for providing the PC9 and PC9/GR cell lines.

\section{References}

1. Jemal A, Siegel R, Xu J and Ward E: Cancer statistics, 2010. CA Cancer J Clin 60: 277-300, 2010.

2. Salomon DS, Brandt R, Ciardiello $F$ and Normanno N: Epidermal growth factor-related peptides and their receptors in human malignancies. Crit Rev Oncol Hematol 19: 183-232, 1995.

3. Ennis BW, Lippman ME and Dickson RB: The EGF receptor system as a target for antitumor therapy. Cancer Invest 9: 553-562, 1991.

4. Lynch TJ, Bell DW, Sordella R, et al: Activating mutations in the epidermal growth factor receptor underlying responsiveness of non-small-cell lung cancer to gefitinib. N Engl J Med 350: 2129-2139, 2004.

5. Maemondo M, Inoue A, Kobayashi K, et al: Gefitinib or chemotherapy for non-small-cell-lung cancer with mutated EGFR. N Engl J Med 362: 2380-2388, 2010.

6. Masago K, Fujita S, Togashi Y, et al: Clinicopathologic factors affecting the progression free survival of patients with advanced non-small-cell lung cancer after gefitinib therapy. Clin Lung Cancer 12: 56-61, 2011.

7. Jackman D, Pao W, Riely GJ, et al: Clinical definition of acquired resistance to epidermal growth factor receptor tyrosine kinase inhibitors in non-small-cell lung cancer. J Clin Oncol 28: 357-360, 2010.

8. Lovly CM and Horn L: Strategies for overcoming EGFR resistance in the treatment of advanced-stage NSCLC. Curr Treat Options Oncol 13: 516-526, 2012.

9. Goldberg SB, Oxnard GR, Digumarthy S, et al: Chemotherapy with erlotinib or chemotherapy alone in advanced non-small cell lung cancer with acquired resistance to EGFR tyrosine kinase inhibitors. Oncologist 18: 1214-1220, 2013.

10. Haddad R, Allen A, Wirth L, Tishler R and Posner M: Integrating novel agents into the curative treatment of head and neck cancer. Expert Rev Anticancer Ther 6: 157-159, 2006.

11. Hanna N, Shepherd FA, Fossella FV, et al: Randomized phase III trial of pemetrexed versus docetaxel in patients with non-small cell lung cancer previously treated with chemotherapy. J Clin Oncol 22: 1589-1597, 2004.

12. Fukuoka M, Yano S, Giaccone G, et al: Multi-institutional randomized phase II trial of gefitinib for previously treated patients with advanced non-small-cell lung cancer (The IDEAL 1 Trial) [corrected]. J Clin Oncol 21: 2237-2246, 2003.

13. Grunwald V and Hidalgo M: Developing inhibitors of the epidermal growth factor receptor for cancer treatment. J Natl Cancer Inst 95: 851-867, 2003.

14. Kim ES, Hirsh V, Mok T, et al: Gefitinib versus docetaxel in previously treated non-small-cell lung cancer (INTEREST): a randomised phase III trial. Lancet 372: 1809-1818, 2008.

15. Steins MB, Reinmuth N, Bischoff H, Kindermann M and Thomas M: Targeting the epidermal growth factor receptor in non-small cell lung cancer. Onkologie 33: 704-709, 2010.

16. Baselga $J$ and Arteaga CL: Critical update and emerging trends in epidermal growth factor receptor targeting in cancer. J Clin Oncol 23: 2445-2459, 2005

17. Herbst RS and Sandler AB: Overview of the current status of human epidermal growth factor receptor inhibitors in lung cancer. Clin Lung Cancer 6 (Suppl 1): S7-S19, 2004.

18. Ciardiello F, Caputo R, Borriello G, et al: ZD1839 (IRESSA), an EGFR-selective tyrosine kinase inhibitor, enhances taxane activity in bcl-2 overexpressing, multidrug-resistant MCF-7 ADR human breast cancer cells. Int J Cancer 98: 463-469, 2002.

19. Park JK, Lee SH, Kang JH, Nishio K, Saijo N and Kuh HJ: Synergistic interaction between gefitinib (Iressa, ZD1839) and paclitaxel against human gastric carcinoma cells. Anticancer Drugs 15: 809-818, 2004.

20. Sirotnak FM, Zakowski MF, Miller VA, Scher HI and Kris MG: Efficacy of cytotoxic agents against human tumor xenografts is markedly enhanced by coadministration of ZD1839 (Iressa), an inhibitor of EGFR tyrosine kinase. Clin Cancer Res 6: 4885-4892, 2000.

21. Shih C, Chen VJ, Gossett LS, et al: LY231514, a pyrrolo[2,3-d] pyrimidine-based antifolate that inhibits multiple folate-requiring enzymes. Cancer Res 57: 1116-1123, 1997.

22. Chattopadhyay S, Moran RG and Goldman ID: Pemetrexed: biochemical and cellular pharmacology, mechanisms, and clinical applications. Mol Cancer Ther 6: 404-417, 2007. 
23. Scagliotti GV, Parikh P, von Pawel J, et al: Phase III study comparing cisplatin plus gemcitabine with cisplatin plus pemetrexed in chemotherapy-naive patients with advanced-stage non-small-cell lung cancer. J Clin Oncol 26: 3543-3551, 2008.

24. Ciuleanu T, Brodowicz T, Zielinski C, et al: Maintenance pemetrexed plus best supportive care versus placebo plus best supportive care for non-small-cell lung cancer: a randomised, double-blind, phase 3 study. Lancet 374: 1432-1440, 2009.

25. Chou TC and Talalay P: Quantitative analysis of dose-effect relationships: the combined effects of multiple drugs or enzyme inhibitors. Adv Enzyme Regul 22: 27-55, 1984.

26. Rinaldi DA, Kuhn JG, Burris HA, et al: A phase I evaluation of multitargeted antifolate (MTA, LY231514), administered every 21 days, utilizing the modified continual reassessment method for dose escalation. Cancer Chemother Pharmacol 44: 372-380, 1999.

27. Thödtmann R, Depenbrock H, Dumez H, et al: Clinical and pharmacokinetic phase I study of multitargeted antifolate (LY231514) in combination with cisplatin. J Clin Oncol 17: 3009-3016, 1999.

28. D'Addario G, Pintilie M, Leighl NB, Feld R, Cerny T and Shepherd FA: Platinum-based versus non-platinum-based chemotherapy in advanced non-small-cell lung cancer: a meta-analysis of the published literature. J Clin Oncol 23: 2926-2936, 2005.

29. Pfister DG, Johnson DH, Azzoli CG, et al: American Society of Clinical Oncology treatment of unresectable non-small-cell lung cancer guideline: update 2003. J Clin Oncol 22: 330-353, 2004.

30. Ardizzoni A and Tiseo M: Second-line chemotherapy in the treatment of advanced non-small cell lung cancer (NSCLC). J Chemother 16 (Suppl 4): 104-107, 2004.

31. Maemondo M: Timing the change of chemotherapy for non small cell lung cancer. Gan To Kagaku Ryoho 39: 1316-1319, 2012 (In Japanese).

32. Oxnard GR, Arcila ME, Chmielecki J, Ladanyi M, Miller VA and Pao W: New strategies in overcoming acquired resistance to epidermal growth factor receptor tyrosine kinase inhibitors in lung cancer. Clin Cancer Res 17: 5530-5537, 2011.
33. VanSchaeybroeck S,Kyula J,Kelly DM,etal:Chemotherapy-induced epidermal growth factor receptor activation determines response to combined gefitinib/chemotherapy treatment in non-small cell lung cancer cells. Mol Cancer Ther 5: 1154-1165, 2006.

34. Li T, Ling YH, Goldman ID and Perez-Soler R: Schedule-dependent cytotoxic synergism of pemetrexed and erlotinib in human non-small cell lung cancer cells. Clin Cancer Res 13: 3413-3422, 2007.

35. Giovannetti E, Lemos C, Tekle C, et al: Molecular mechanisms underlying the synergistic interaction of erlotinib, an epidermal growth factor receptor tyrosine kinase inhibitor, with the multitargeted antifolate pemetrexed in non-small-cell lung cancer cells. Mol Pharmacol 73: 1290-1300, 2008

36. Davies AM, Ho C, Lara PN Jr, Mack P, Gumerlock PH and Gandara DR: Pharmacodynamic separation of epidermal growth factor receptor tyrosine kinase inhibitors and chemotherapy in non-small-cell lung cancer. Clin Lung Cancer 7: 385-388, 2006.

37. Ruvolo PP, Deng X and May WS: Phosphorylation of Bcl-2 and regulation of apoptosis. Leukemia 15: 515-522, 2001.

38. Diel P, Smolnikar K and Michna H: The pure antiestrogen ICI 182780 is more effective in the induction of apoptosis and down regulation of BCL-2 than tamoxifen in MCF-7 cells. Breast Cancer Res Treat 58: 87-97, 1999.

39. Sordella R, Bell DW, Haber DA and Settleman J: Gefitinibsensitizing EGFR mutations in lung cancer activate anti-apoptotic pathways. Science 305: 1163-1167, 2004.

40. Amann J, Kalyankrishna S, Massion PP, et al: Aberrant epidermal growth factor receptor signaling and enhanced sensitivity to EGFR inhibitors in lung cancer. Cancer Res 65: 226-235, 2005.

41. Van Schaeybroeck S, Karaiskou-McCaul A, Kelly D, et al: EGFR activity determines response of colorectal cancer cells to gefitinib (Iressa) alone and in combination with chemotherapy. Clin Cancer Res 11: 7480-7489, 2005. 